\title{
Spectral Properties of Second Order Differential Equations with Spectral Parameter in the Boundary Conditions
}

\author{
Rostam K. Saeed ${ }^{1, *}$, Karwan H. F. Jwamer ${ }^{2, *}$ and Khelan H. Qader ${ }^{2}$ \\ ${ }^{1}$ Department of Mathematics, College of Science, University of Salahddin, Kurdistan, Region-Erbil, Iraq \\ ${ }^{2}$ Departmen of Mathematics, Faculty of Science and Science Education-School, University of Sulaimani, Kurdistan, Region-Sulaimani, \\ Iraq
}

Received: 16 Jun. 2013, Revised: 12 Oct. 2013, Accepted: 13 Oct. 2013

Published online: 1 Jan. 2014

Abstract: In this paper, we found the location and asymptotic of the eigenvalues of the linear differential equation

$$
-y^{\prime \prime}+q(x) y=\lambda^{2} p(x) y, x \in(0, a)
$$

with the boundary conditions $y^{\prime}(a)+i \lambda y(a)=y^{\prime}(0)+i \lambda y(0)=0$ when $\rho(x)>0$ and the normalized condition $\int_{0}^{a} \rho(x)|y(x)|^{2} d x=1$, where $\lambda$ is a spectral parameter.

Keywords: boundary conditions, eigenvalues, eigenfunctions, asymptotic behaviors.

\section{Introduction}

Consider the linear differential equation

$$
-y^{\prime \prime}+q(x) y=\lambda^{2} p(x) y, x \in(0, a)
$$

where $\lambda$ is a spectral parameter, $q(x)$ and $p(x)$ are realvalued continuous functions and positive on the interval $(0, a)$ with the boundary conditions contains the spectral parameter $\lambda$ and normalized condition of the forms:

$$
\begin{gathered}
y^{\prime}(a)+i \lambda y(a)=y^{\prime}(0)+i \lambda y(0)=0 \\
\int_{0}^{a} \rho(x)|y(x)|^{2} d x=1
\end{gathered}
$$

Numerous problems of oscillation theory for spatially distributed systems lead to necessity of study of eigenvalues and their appropriate eigenfunctions of differential operators as well as to issues related to study of various functional of eigenvalues and eigenfunctions. It is known that many problems of mathematical physics, mechanics, elasticity theory, optimal control leads to the problem of studying the spectrum of differential operators and the expansion of arbitrary functions in series of eigenfunctions of the operator. In [1-6], the eigenvalues and the corresponding eigenfunctions to the differential (1) studied, but they used different boundary conditions in which we used in this paper.

\section{The main results}

The aim of our article is to found the location and asymptotic for the eigenvalues to the problem (1)-(3) (named problem $H_{1}$ ) which appear in the following theorems in below:

Theorem 1: Let $\lambda$ be an eigenvalue corresponding to the eigenfunction $y(x)$ of the problem $H_{1}$, if $\delta \neq 0$ and $\rho(x)>0$ then $\lambda$ is complex, and located in upper half plane.

Proof:

Multiply equation (1) by $\bar{y}(x)$ and integrate the resulting

\footnotetext{
*Corresponding author e-mail: rostamkarim64@uni-sci.org, jwameri1973@gmail.com
} 
equation from 0 up to $a$, as a result we shall get:

$$
\begin{aligned}
& \int_{0}^{a} y^{\prime \prime}(x) \bar{y}(x) d x+\int_{0}^{a} q(x) y(x) \bar{y}(x) d x \\
& =\int_{0}^{a} \lambda^{2} \rho(x) y(x) \bar{y}(x) d x, \\
& \left.-y^{\prime}(x) \bar{y}(x)\right]_{0}^{a}+\int_{0}^{a}\left|y^{\prime}(x)\right|^{2}+\int_{0}^{a} q(x)\left|y^{\prime}(x)\right|^{2} d x \\
& =\int_{0}^{a} \lambda^{2} \rho(x) y(x) \bar{y}(x) d x, \\
& -y^{\prime}(a) \bar{y}(a)+y^{\prime}(0) \bar{y}(0) \int_{0}^{a}\left|y^{\prime}(x)\right|^{2}+\int_{0}^{a} q(x)\left|y^{\prime}(x)\right|^{2} d x \\
& =\int_{0}^{a} \lambda^{2} \rho(x) y(x) \bar{y}(x) d x,
\end{aligned}
$$

In view of boundary conditions(2) we have:

$$
\begin{aligned}
& -i \lambda y^{\prime}(a) \bar{y}(a)-i \lambda y^{\prime}(0) \bar{y}(0) \int_{0}^{a}\left|y^{\prime}(x)\right|^{2}+\int_{0}^{a} q(x)\left|y^{\prime}(x)\right|^{2} d x \\
& =\int_{0}^{a} \lambda^{2} \rho(x) y(x) \bar{y}(x) d x,
\end{aligned}
$$

Hence

$$
\begin{aligned}
& -i \lambda|y(a)|^{2}-i \lambda|y(0)|^{2}+\int_{0}^{a}\left|y^{\prime}(x)\right|^{2}+\int_{0}^{a} q(x)\left|y^{\prime}(x)\right|^{2} d x \\
& =\int_{0}^{a} \lambda^{2} \rho(x) y(x) \bar{y}(x) d x .
\end{aligned}
$$

In equation (1) and in boundary condition (2) replace $\mathrm{y}(\mathrm{x})$ by $\bar{y}(x)$ the following equations are obtained:

$-\overline{y^{\prime \prime}}+q(x) \bar{y}(x)=\lambda^{2} p(x) \bar{y}(x)$,

$\overline{y^{\prime}}(0)=\bar{y}(a)+i \lambda \bar{y}(a)=0$.

Multiplying differential equation on $y(x)$ and integrate from 0 up to a, yields:

$$
\begin{aligned}
& i \lambda y^{\prime}(a) \bar{y}(a)+i \lambda y^{\prime}(0) \bar{y}(0) \int_{0}^{a}\left|y^{\prime}(x)\right|^{2}+\int_{0}^{a} q(x)\left|y^{\prime}(x)\right|^{2} d x \\
& =\int_{0}^{a} \lambda^{2} \rho(x) y(x) \bar{y}(x) d x
\end{aligned}
$$

Subtracting equations (4) from (5) we obtain:

$i(\lambda+\bar{\lambda})\left(|y(a)|^{2}+|y(0)|^{2}\right)=\left(\lambda^{2}-\left(\bar{\lambda}^{2}\right) \int_{0}^{a} \rho(x)|y(x)|^{2} d x\right.$, $i(\lambda+\bar{\lambda})\left(|y(a)|^{2}+|y(0)|^{2}\right)=\left(\lambda-(\bar{\lambda}) \int_{0}^{a} \rho(x)|y(x)|^{2} d x\right.$,

Clearly, $(\lambda+\bar{\lambda}) \neq 0$ because $\delta \neq 0$, then

$$
\begin{aligned}
& (\lambda-\bar{\lambda}) \int_{0}^{a}(x)|y(x)|^{2} d x-i\left(|y(a)|^{2}+|y(0)|^{2}\right)=0, \\
& 2 i \sigma \int_{0}^{a}(x)|y(x)|^{2} d x-i\left(|y(a)|^{2}+|y(0)|^{2}\right)=0 .
\end{aligned}
$$

Thus, $\sigma=\frac{|y(a)|^{2}+|y(0)|^{2}}{2 \int_{0}^{a}(x)|y(x)|^{2} d x}$,

and since $2 \int_{0}^{a}(x)|y(x)|^{2} d x=1$, then $\sigma>0$.
Hence $\lambda$ is complex and located in upper half plane. Thus the theorem is proved.

\section{Theorem 2:}

Asymptotic behaviour of eigenvalues of the problem (1)-(2) in the case of regular and in the sector $T_{1}$ has the form:

$$
\begin{aligned}
& \lambda_{m}=\frac{1}{d}\left(m \pi-\frac{i}{2} \ln C_{0}+O\left(\frac{1}{m}\right)\right), \\
& \text { where } C_{0}=\frac{-(\sqrt{\rho(a)}+1)(1-\sqrt{\rho(0)})}{\sqrt{\rho(a)}-1)(1+\sqrt{\rho(0)})}
\end{aligned}
$$

Proof:

Consider the determinant of $\Delta(\lambda)$, defined by

$$
\begin{aligned}
& \Delta(\lambda)=\left|U_{k}\left(y_{j}\right)\right|_{k, j=0,1} . \\
& U_{k}\left(\tilde{y}_{j}\right)=\sum_{r}^{2}=1\left(-i \lambda w_{k}\right)^{2-r} \tilde{y}_{j}^{(r-1)}(a, \lambda)=0 \\
& , k=0, j=0,1 . \\
& U_{k}\left(\tilde{y}_{j}\right)=\sum_{r}^{2}=1\left(-i \lambda w_{k}\right)^{2-r} \tilde{y}_{j}^{(r-1)}(0, \lambda)=0 \\
& , k=1, j=0,1 . \\
& U_{0}\left(\tilde{y}_{0}\right)=(-i \lambda) \tilde{y}_{0}(a, \lambda)+\tilde{y}_{0}(a, \lambda)=0 . \\
& U_{0}\left(\tilde{y}_{1}\right)=(-i \lambda) \tilde{y}_{1}(a, \lambda)+\tilde{y}_{1}(a, \lambda)=0 . \\
& U_{0}\left(\tilde{y}_{0}\right)=i \lambda \tilde{y}_{0}(0, \lambda)+\tilde{y}_{1}(0, \lambda)=0 . \\
& U_{0}\left(\tilde{y}_{0}\right)=i \lambda \tilde{y}_{1}(0, \lambda)+\tilde{y}_{1}(0, \lambda)=0 . \\
& y_{k}^{(s)}(x, \lambda)=\left(\varphi_{k} \lambda\right)^{s} e^{\lambda \int_{0}^{a} \varphi_{k} d x}\left[A_{0}+O\left(\frac{1}{\lambda}\right)\right], \\
& s=0,1 .
\end{aligned}
$$

The following results can be obtained by using the formula (6) and the boundary conditions (2).

$$
\begin{aligned}
& U_{0}\left(\tilde{y_{0}}\right)=\left(i w_{0}^{\prime} \lambda\right)\left[\frac{1}{\sqrt[4]{\rho(0)}}\right](\sqrt{\rho(0)}-1) \\
& =i \lambda e^{i \lambda d}\left(\sqrt{\rho(a)-1)}\left[\frac{1}{\sqrt[4]{\rho(0)}}\right]\right.
\end{aligned}
$$

where $w_{j}^{\prime}=e^{i\left(\frac{(j-k) \pi}{n}\right)}$.

$$
\begin{aligned}
& U_{0}\left(\tilde{y_{1}}\right)=-i \lambda e^{-i \lambda d)}(\sqrt{\rho(a)}+1)\left[\frac{1}{\sqrt[4]{\rho(0)}}\right] \\
& U_{1}\left(\tilde{y_{0}}\right)=i \lambda(1-\sqrt{\rho(0)})\left[\frac{1}{\sqrt[4]{\rho(0)}}\right] \\
& U_{1}\left(\tilde{y_{1}}=i \lambda(1+\sqrt{\rho(0)})\left[\frac{1}{\sqrt[4]{\rho(0)}}\right]\right.
\end{aligned}
$$


$\Delta(\lambda)=\left\{\begin{array}{l}i \lambda e^{i \lambda d)}(\sqrt{\rho(a)}-1)\left[\frac{1}{\sqrt[4]{\rho(0)}}\right]-i \lambda e^{-i \lambda d)}(\sqrt{\rho(a)}+1) \\ {\left[\frac{1}{\sqrt[4]{\rho(0)}}\right]} \\ i \lambda(1-\sqrt{\rho(0)})\left[\frac{1}{\sqrt[4]{\rho(0)}}\right] i \lambda(1+\sqrt{\rho(0)})\left[\frac{1}{\sqrt[4]{\rho(0)}}\right] \\ =0\end{array}\right.$

and

$\Delta(\lambda)=\left|\begin{array}{l}(i \lambda)^{2} e^{i \lambda d(1+\sqrt{\rho(0)}(\sqrt{\rho(a)-1)}}\left[\frac{1}{\sqrt[4]{\rho(0)}}\right] \\ {\left[\frac{1}{\sqrt[4]{\rho(a)}}\right]+(i \lambda)^{2} e^{-i \lambda d(1-\sqrt{\rho(0)}(\sqrt{\rho(a)+1)}}} \\ {\left[\frac{1}{\sqrt[4]{\rho(0)}}\right]\left[\frac{1}{\sqrt[4]{\rho(a)}}\right]} \\ =0\end{array}\right|$

Suppose $f(\lambda)=(i \lambda)^{2}\left[\frac{1}{\sqrt[4]{\rho(0)}}\right]\left[\frac{1}{\sqrt[4]{\rho(a)}}\right]$

$\Delta(\lambda)=f(\lambda)\left[\left(1+\sqrt{\rho(0)}\left(\sqrt{\rho(a)-1)} e^{i \lambda d}+\right.\right.\right.$

$\left(1-\sqrt{\rho(0)}\left(\sqrt{\rho(a)+1)} e^{-i \lambda d}\right]=0\right.$

$$
e^{2 i \lambda d}=\frac{-(1+\sqrt{\rho(0)}(\sqrt{\rho(a)-1)}}{(1-\sqrt{\rho(0)}(\sqrt{\rho(a)+1)}}
$$

where

$C_{0}=\frac{-(1+\sqrt{\rho(0)}(\sqrt{\rho(a)-1)}}{(1-\sqrt{\rho(0)}(\sqrt{\rho(a)+1)}}$

$e^{2 i \lambda d}=C_{0} \rightarrow 2 i \lambda d=\ln C_{40}+2 m \pi i+o\left(\frac{1}{m}\right)$

$\lambda_{m}=\frac{1}{d}\left(m \pi \pi-\frac{i}{2} \ln C_{0}+o\left(\frac{1}{m}\right)\right)$.

In the case of regular the sector $T_{1}$ asymptotic behaviour of spectrum has the form:

$\lambda_{m}=\frac{1}{d}\left(m \pi \pi-\frac{i}{2} \ln C_{0}+o\left(\frac{1}{m}\right)\right)$, where $m=N, N+1, \ldots$

( $\mathrm{N}$ is natural number), and in the sector $T_{2}$

$\lambda_{m}=\frac{1}{d}\left(m \pi \pi+\frac{i}{2} \ln C_{0}+o\left(\frac{1}{m}\right)\right)$, where $m=N, N+1, \ldots$

( $\mathrm{N}$ is natural number). Thus the theorem is proved.

Theorem 3: Asymptotic behavior of eigenvalues of the problem (1)-(2) in the case of irregular and in the sector $T_{1}$ has the form: $\lambda_{m}=\frac{1}{d}\left(-m \pi+\frac{i}{2} \ln C_{0}+\frac{i}{2} \ln \lambda^{0}+o(1)\right.$ and in the sector $T_{2}$ asymptotic behaviour of spectrum has the form:

$\lambda_{m}=\frac{1}{d}\left(-m \pi-\frac{i}{2} \ln C_{0}-\frac{i}{2} \ln \lambda^{0}+o(1) \quad\right.$ where $C_{0}=\frac{(4 i)^{2}}{q(a)+\frac{1}{4} \rho^{n}(a)}$ and $d=\int_{0}^{a} \sqrt{\rho(x)} d x$, such that $q(a)+\frac{1}{4} \rho^{\prime \prime}(a) \neq 0 . q(0)+\frac{1}{4} \rho^{\prime \prime}(0) \neq 0$.
Proof:

Consider the determinant of $\Delta(\lambda)$, which is defined by:

$$
\begin{aligned}
& \Delta(\lambda)=\left|U_{k}\left(y_{j}\right)\right|_{(k, j=0,1)}, \\
& U_{k}\left(\tilde{y_{j}}\right)=\left(-i \lambda w_{k}\right) \tilde{y_{j}}(a, \lambda)+\tilde{y_{j}}(a, \lambda)=0, \text { for } k=0, \\
& U_{k}\left(\tilde{y_{j}}\right)=\left(-i \lambda w_{k}\right) \tilde{y_{j}}(0, \lambda)+\tilde{y_{j}}(0, \lambda)=0, \text { for } k=1, \\
& U_{0}\left(\tilde{y_{0}}\right)=-i \lambda \tilde{y_{0}}(a, \lambda)+\tilde{y_{0}}(a, \lambda), \\
& U_{0}\left(\tilde{y_{1}}\right)=-i \lambda \tilde{y_{1}}(a, \lambda)+\tilde{y_{1}}(a, \lambda), \\
& U_{1}\left(\tilde{y_{0}}\right)=i \lambda \tilde{y_{0}}(0, \lambda)+\tilde{y_{0}}(0, \lambda), \\
& U_{1}\left(\tilde{y_{1}}\right)=i \lambda \tilde{y_{1}}(0, \lambda)+\tilde{y_{1}}(0, \lambda), \\
& U_{0}\left(y_{0}\right)=e^{i \lambda d}(-i \lambda)\left[s_{1}\left(A_{0(p)}(a)+s_{3}\right)+\right. \\
& \left.\frac{1}{\lambda} \frac{1}{2 i} A_{1(p)}(a)+s_{4}+O\left(1 \frac{1}{\lambda^{2}}\right)\right], \\
& U_{0}\left(y_{1}\right)=e^{i \lambda d}(-i \lambda)\left[s_{1}\left(A_{0(p)}(a)+s_{3}\right)+\right. \\
& \left.\frac{1}{\lambda} \frac{1}{2 i} A_{0(p)}(a)+s_{4}+O\left(1 \frac{1}{\lambda^{2}}\right)\right], \\
& U_{1}\left(y_{0}\right)=i \lambda\left[s_{2}\left(A_{0(p)}(0)+s_{3}\right)+\right. \\
& \left.2 \frac{1}{\lambda} \frac{1}{2 i} A_{0(p)}(a)+s_{4}+O\left(\frac{1}{\lambda^{2}}\right)\right], \\
& U_{1}\left(y_{1}\right)=i \lambda\left[s_{1}\left(A_{0(p)}(0)+s_{3}\right)-\right. \\
& \left.2 \frac{1}{\lambda} \frac{1}{-2 i} A_{0(p)}(a)-s_{5}+O\left(\frac{1}{\lambda^{2}}\right)\right],
\end{aligned}
$$

where

$$
\begin{aligned}
A_{0(p)}(a) & =1 \\
A_{1(p)}(a) & =\int_{0}^{a}\left(q(t) A_{0}-A_{0}^{\prime \prime}\right) A_{0} d t, \ldots, \\
A_{n(p)}(a) & =\int_{0}^{a}\left(q(t) A_{n-1}-A_{n-1}^{\prime \prime}\right) A_{0} d t, \ldots, \\
s_{1} & =(1+\sqrt{(\rho(a)}), \\
s_{2} & =(1+\sqrt{\rho(a)})), \\
s_{3} & =\frac{1}{\lambda} \frac{1}{2 i} A_{1(p)}(a) . \\
s_{4} & =\frac{21}{\lambda^{2}} \frac{1}{(2 i)^{2}} A_{1(p)}^{\prime}(a)
\end{aligned}
$$

and

$$
s_{5} \quad=\frac{21}{\lambda^{2}} \frac{1}{(2 i)^{2}} A_{1(p)}^{\prime}(0)
$$

Spectrum of (1)-(2) coincides with the set of roots of the equation: $\Delta(\lambda)=0$, then

$$
\begin{aligned}
& \Delta(\lambda)=\left|\begin{array}{ll}
a_{11} & a_{12} \\
a_{21} & a_{22}
\end{array}\right|=0 \text { Where } \\
& a_{11}= e^{i \lambda d}(-i \lambda)\left(s_{1}\left(A_{0(p)}(a)+\frac{1}{\lambda} \frac{1}{2 i} A_{1(p)}(a)\right)\right. \\
&\left.+2 \frac{1}{2} \frac{1}{2 i} A_{0(p)}^{\prime}(a)+s_{4}+O\left(\frac{1}{\lambda^{2}}\right)\right),
\end{aligned}
$$




$$
\begin{aligned}
& a_{12}=e^{i \lambda d}(-i \lambda)\left(s_{1}\left(A_{0(p)}(a)-s_{3}\right)\right. \\
& \left.\left.-2 \frac{1}{\lambda} \frac{1}{(-2 i)}\right) A_{0(p)}^{\prime}(a)-s_{4}+O\left(\frac{1}{\lambda^{2}}\right)\right), \\
& a_{21}=i \lambda\left(s_{2}\left(A_{0(p)}(0)+\frac{1}{\lambda} \frac{2}{2 i} A_{1(p)}(0)\right)\right. \\
& \left.+\frac{21}{\lambda} \frac{2}{2 i} A_{0(p)}^{\prime}(0)+s_{5}+O\left(\frac{1}{\lambda^{2}}\right)\right) \\
& a_{22}=i \lambda\left(s_{2}\left(A_{0(p)}(0)+\frac{1}{\lambda} \frac{1}{2 i} A_{1(p)}(0)\right)\right. \\
& \left.\left.-2 \frac{1}{\lambda} \frac{1}{-2 i}\right) A_{0(p)}^{\prime}(0)-s_{5}+O\left(\frac{1}{\lambda^{2}}\right)\right), \\
& \Longrightarrow-(i \lambda)^{2} e^{i \lambda d}\left(s_{1}\left(A_{0(p)}(a)+s_{3}\right)\right. \\
& \left.+2 \frac{1}{\lambda} \frac{2}{2 i} A_{0(p)}^{\prime}(a)+s_{4}+O\left(\frac{1}{\lambda^{2}}\right)\right) x\left(s _ { 2 } \left(A_{0(p)}(0)\right.\right. \\
& \left.\left.\left.+\frac{1}{\lambda} \frac{1}{-2 i)}\right) A_{1(p)}(0)\right)-2 \frac{1}{\lambda} \frac{1}{(-2 i)}\right) A_{0(p)}^{\prime}(0)-s_{5} \\
& \left.+O\left(\frac{1}{\lambda^{2}}\right)\right)+(i \lambda)^{2} e^{(-i \lambda d)}\left(s_{1}\left(A_{0(p)}(a)-s_{3}\right)\right. \\
& \left.\left.-2 \frac{1}{\lambda} \frac{1}{(-2 i)}\right) A_{0(p)}^{\prime}(a)-s_{4}+O\left(\frac{1}{\lambda^{2}}\right)\right) \\
& +\left(s_{2}\left(A_{0(p)}(0)+\frac{1}{\lambda} \frac{1}{2 i} A_{1(p)}(0)\right)\right. \\
& \left.\left.-2 \frac{1}{\lambda} \frac{1}{(-2 i)}\right) A_{0(p)}^{\prime}(0)-s_{5}+O\left(\frac{1}{\lambda^{2}}\right)\right)=0,
\end{aligned}
$$

$$
\begin{aligned}
& e^{2 i \lambda d} \\
& =\frac{\left.\left(-4 \lambda^{2}+2 \frac{\lambda}{i} A_{(1(p)}\right)(a)-A_{1(p)}^{\prime}(a)\right)\left(4 \lambda^{2}+A_{1(p)}^{\prime}(0)\right)}{\left(q(a)+\frac{1}{4} \rho^{\prime \prime}(a)\right)\left(q(0)+\frac{1}{4} \rho^{\prime \prime}(0)\right)[1]} \\
& =0,
\end{aligned}
$$

From which we obtain that:

$$
\begin{aligned}
& e^{2 i \lambda d} \\
& =\frac{-16 \lambda^{4}}{\left(q(a)+\frac{1}{4} \rho^{\prime \prime}(a)\right)\left(q(0)+\frac{1}{4} \rho^{\prime \prime}(0)\right)[1]} \\
& =0, \\
& \Longrightarrow e^{2 i \lambda d}[1]=C_{0} \cdot \lambda^{4}
\end{aligned}
$$

where

$$
C_{0}=\frac{(4 i)^{2}}{\left(q(a)+\frac{1}{4} \rho^{\prime \prime}(a)\right)\left(q(0)+\frac{1}{4} \rho^{\prime \prime}(0)\right)[1]}=0
$$

and $q(a)+\frac{1}{4} \rho^{\prime \prime}(a) \neq 0, q(0)+\frac{1}{4} \rho^{\prime \prime}(0) \neq 0$.

Taking the initial approximation $\lambda_{0}=\frac{-m \pi}{d}$, and using the method of successive approximation we obtain: $\operatorname{lambda} a_{m}=\frac{1}{d}\left(m \pi-\frac{i}{2} \ln C_{0}-\frac{i}{2} \ln \pi^{4}+o(1), \quad\right.$ where $m=N, N+1, \ldots(\mathrm{N}$ is natural number $)$, and in the sector $T_{2} \quad l a m b d a_{m}=\frac{1}{d}\left(m \pi+\frac{i}{2} \ln C_{0}+\frac{i}{2} \ln \lambda^{2}+o(1) \quad\right.$ where $m=N, N+1, \ldots(\mathrm{N}$ is natural number $)$. Thus the theorem is proved.

\section{References}

[1] G. A. Aigounov , K. H-F. Jwamer and G.A. Djalaeva, Estimates for the eigenfunctions of the Regge problem, Mat. Zametki, 92,141-144 (2012).

[2] M. G. Crane, About return problems for non uniform structure, DSC USSR, 76, 345-348 (1951).

[3] M. G. Crane , Test of density of non uniform symmetric strings on spectrum of its frequencies, DSC USSR, 82, 669672 (1952).

[4] K. H-F. Jwamer and G. A. Aigounov, About Uniform Limitation of Normalized Eigen Functions of T. Regge Problem in the Case of Weight Functions, Satisfying to Lipschitz Condition, Gen. Math. Notes, 1, 115-129 (2010).

[5] K. H-F. Jwamer and K. H. Qader, Estimates Normalized Eigenfunction to the Boundary Value Problem in Different Cases of Weight Functions, Int. J. Open Problems Compt. Math., 4, 28-38 (2011).

[6] Ya. D. Tamarkin, About some general problems of theory of ordinary linear Differential equations and about decomposition of arbitrary functions in series, Petrograd, (1917). 


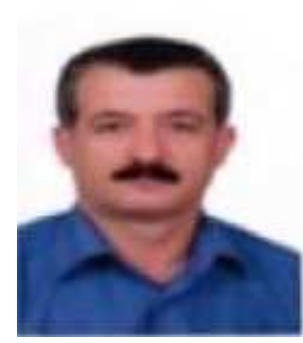

Rostam K. Saeed
$\begin{aligned} & \text { Professor of Mathematics } \\ & \text { (Numerical }\end{aligned}$
$\begin{aligned} & \text { Department of Mathematics, } \\ & \text { College of Science, }\end{aligned}$
University of Salahaddin/Erbil,
Iraq. He Awarded PhD
in Mathematics (Numerical
Analysis) from Salahaddin
University, Department of

Mathematics, College of Science. He supervised nine M.Sc. students and two $\mathrm{PhD}$ students in Department of Mathematics, College of Science, University of Salahaddin / Erbil, University of Sulaimani and Dohuk University, Iraq. His researches interests include numerical solution of integral and integro-differential equations, approximation by spline functions .



Karwan H. F. Jwamer Assistant Professor of Mathematics Department of Faculty of Science and Science Education - School of Science at the Sulaimani University, Kurdistan Region, Sulaimani,Iraq. He obtained Ph.D from Dagestan State University, South of Russian in 2010. His researches interests include spectral analysis for different type boundary value problems, approximation by spline functions. He supervised three students one Ph.D and two M.Sc. in the field of differential equations and numerical analysis. He has published over forty six papers in these areas. He is referee and editorial board for more than sixteen mathematical journals.
Khelan H. Qader assistant lecture of Mathematics Department of Faculty of Science and Science Education - School of Science at the Sulaimani University, Kurdistan Region, Sulaimani,Iraq. Received her B.Sc. and M.Sc. degree in Mathematics in 2007 and 2011 from Sulaimani University. Her researches interests in the field of spectral analysis for boundary value problems contains spectral parameters. 